\title{
Reduction in treatment delay by paramedic ECG diagnosis of myocardial infarction with direct CCU admission
}

\author{
M W Millar-Craig, A V Joy, M Adamowicz, R Furber, B Thomas
}

\begin{abstract}
Objectives-To establish the feasibility of training paramedics to diagnose acute myocardial infarction by ECG before hospital admission and whether direct paramedic coronary care admission, arranged by very high frequency (VHF) radio communication with the coronary care unit (CCU), would reduce delay of thrombolysis treatment.

Design-Prospective controlled study.

Setting-District general hospital CCU and a local district ambulance paramedic service.

Patients-124 patients with ECG evidence of myocardial infarction or ischaemia admitted directly to the CCU by the paramedic service were compared with 123 patients admitted by the emergency department and subsequently transferred to the CCU.
\end{abstract}

Main outcome measures-ECG diagnostic accuracy by paramedics, and interval durations for CCU admission and thrombolysis.

Results-ECG diagnostic accuracy by the paramedics was $87.5 \%$ in the training phase and $92 \%$ in admission. The total call to thrombolysis interval was reduced from 154 to 93 minutes and the "door to needle" interval was reduced from 97 to 37 minutes.

Department of

Cardiology, Derbyshire Royal Infirmary NHS Trust, Derby

M W Millar-Craig A V Joy

Coronary Care Unit M Adamowicz

FASI, Corporate Planning, Derbyshire Ambulance Service Trust, Kingsway, Derby

R Furber

IHSM, Training Support

B Thomas

Correspondence to: Dr M W Millar-Craig, Department of Cardiology, Derbyshire Royal Infirmary NHS Trust, London Road, Derby DE1 2QY, UK.

Accepted for publication 4 July 1997 contacting the emergency services (or general practitioner) and arriving in hospital ("call to arrival" time), and between their arrival in hospital and administration of thrombolytic treatment ("door to needle" time). The call to arrival delay for patients contacting the emergency services directly averaged 46 minutes for eight American cities ${ }^{5}$ and 41 minutes for six British centres. ${ }^{6}$ The door to needle time averaged 84 minutes in the American study and 80 minutes in the British study. Patients in the British study who called and were seen by their general practitioner had a call to arrival time of 90 minutes.

An audit at Derbyshire Royal Infirmary in 1989 showed very similar intervals, with a prehospital interval (onset of symptoms to arrival) of 182 minutes for emergency service admissions, a call to arrival interval of 291 minutes for patients admitted by their general practitioner, and a door to needle interval of 90 minutes. ${ }^{7}$ Most patients were admitted by the emergency department, where a diagnosis of myocardial infarction was confirmed, and subsequently transferred to the coronary care unit (CCU) for thrombolytic treatment.

Various methods have been considered to reduce the interval between the initial hospital call and thrombolysis. Encouraging results were obtained in the Grampian region early anistreplase trial, ${ }^{8}$ in which myocardial infarction was diagnosed and treated with anistreplase at home by general practitioners before transfer to hospital, but the larger multicentre European myocardial infarction project group ${ }^{9}$ and myocardial infarction triage and intervention studies ${ }^{10}$ had less positive results. Administration of thrombolytic treatment in the emergency department after ECG documentation of myocardial infarction may reduce the interval duration, ${ }^{11}$ but has logistic implications. The development of a fast track within the emergency department ${ }^{12}$ may allow faster diagnosis and treatment of myocardial infarction but in one study it was associated with a false positive diagnosis and inappropriate use of thrombolytic treatment in $24 \%$ of patients. ${ }^{13}$ Alternatively, a paramedic team incorporating a physician can also be used to diagnose and treat myocardial infarction by thrombolysis before transfer to hospital ${ }^{1415}$ with less delay in treatment.

Although the concept of a mobile CCU was initiated in the UK by the Belfast group in $1967,{ }^{16}$ this was a physician led team attached to the cardiac unit. The use of highly trained ambulance attendants was first initiated in 
Brighton in $1973 .{ }^{17}$ These personnel were trained to recognise and treat complications of myocardial infarction, including ventricular fibrillation, and acted independently with no immediate help from medical or nursing staff. The success of this unit ${ }^{18}$ has been followed by the development of highly trained ambulance paramedic units in many centres over the past 10 years. In some centres ${ }^{19}{ }^{20}$ this involved the recording of a 12 lead ECG, which was transmitted by cellular telephone to a base physician who either gave instruction for thrombolysis before transportation, or arranged immediate treatment on arrival.

A logical development of precoronary care management of myocardial infarction would be the training of paramedics to record and interpret 12 lead ECGs for patients with suspected myocardial infarction, with transfer directly to a CCU for immediate thrombolytic treatment if there is evidence of myocardial infarction. This would bypass any delay in the emergency department as well as alert coronary care staff, before admission, of the imminent arrival of a patient who may require thrombolysis. This method was the basis for the present study that was designed to reduce the interval between the patient's call for help and initiation of thrombolytic treatment.

The primary objectives of this study were to establish the feasibility of training paramedics to diagnose myocardial infarction by ECG and the potential benefit of direct CCU admission arranged by the paramedics of patients with ECG evidence of infarction or ischaemia, as assessed by the time intervals between the initial call and coronary care arrival and between arrival and thrombolysis. During an initial training phase (phase I), baseline data were obtained on 120 coronary care patients who were admitted to the accident and emergency department and received standard management. The second phase (phase II) evaluated outcome in a further group of 120 patients with suspected acute myocardial infarction or acute myocardial ischaemia admitted directly to coronary care by the paramedics.

Admission of patients with evidence of myocardial infarction was arranged by very high frequency (VHF) radio communication directly with the CCU, thus reducing delay associated with the telephone system and the hospital admissions service. A prospective study was designed to establish whether such a scheme could achieve a significant reduction in interval duration.

\section{Patients and methods}

Following approval by the local ambulance paramedic steering committee and the southern Derbyshire ethics committee, selected qualified paramedics were invited to participate in a new programme involving the recording and interpretation of standard 12 lead ECGs in patients with suspected myocardial infarction before their transfer to hospital.

Candidates for standard paramedic training must be qualified accident and emergency technicians, with at least two years post-basic experience. They take an entrance examination and then undertake a three stage course of study to qualify. They are required to demonstrate their knowledge and proficiency by written examination and practical assessment.

All fully qualified paramedics from five ambulance stations in southern Derbyshire that normally admitted patients to the Derbyshire Royal Infirmary participated in the study. Stations that normally admitted to other hospitals were not included.

Training for the study was specific and structured. At an initial session the nature and aims of the project were explained and more detailed instruction given on clinical aspects of acute myocardial infarction. Practical training in 12 lead ECG recording was given, and this was followed by training in recognition of acute myocardial infarction, bundle branch block, chronic myocardial infarction, and other electrocardiographic changes. Participants then underwent a day of hospital based training in the cardiothoracic measurement department, followed by a one day attachment to the CCU.

Phase I started when the initial teaching had been completed. During phase I, the training phase of the study, paramedics were instructed to record and report on a 12 lead ECG using a standard paramedic clinical data form when called by the emergency service to a patient with suspected myocardial infarction. The patient was then taken to the Derbyshire Royal Infirmary accident and emergency department, in the usual way, for appropriate medical management, which would involve transfer to the CCU and thrombolytic treatment at the discretion of the medical staff. ECGs recorded by the paramedics in phase I were not presented to the admitting medical staff but were filed separately by the study coordinator who maintained a detailed audit of interval durations and copies of the paramedics' clinical data forms. The ECGs were subsequently examined by a cardiologist and graded for technical quality and accuracy of interpretation. Paramedics developed their ECG recording and interpretation skills during this phase, and baseline data were obtained for call to hospital and door to needle intervals. Regular reviews of ECG interpretation were held with the paramedics. The ECG machines used in the study were the DMS 730 (Simonsen and Weel, Sidcup, Kent, UK) and the Lifepak 10 (Physio Control, Basingstoke, UK). The Lifepak machines were available to the ambulance service for the duration of the study. The MAC PC (Marquette Electronics Ltd, Manchester, UK) was also available for a trial period of three months during the study.

Individual paramedics who had recorded at least 10 ECGs of acceptable quality and with an interpretation accuracy of $80 \%$ were allowed to proceed to the "active" phase II of the study. Before phase II, a VHF radio transmitter/receiver was installed in the CCU, the coronary care nursing staff received training in radio communication, and all admitting medical staff were informed of the study. During phase II, paramedics recorded and interpreted a 12 lead ECG at the site of the call and, if it showed evidence of myocardial infarction or ischaemia, were authorised to admit the 


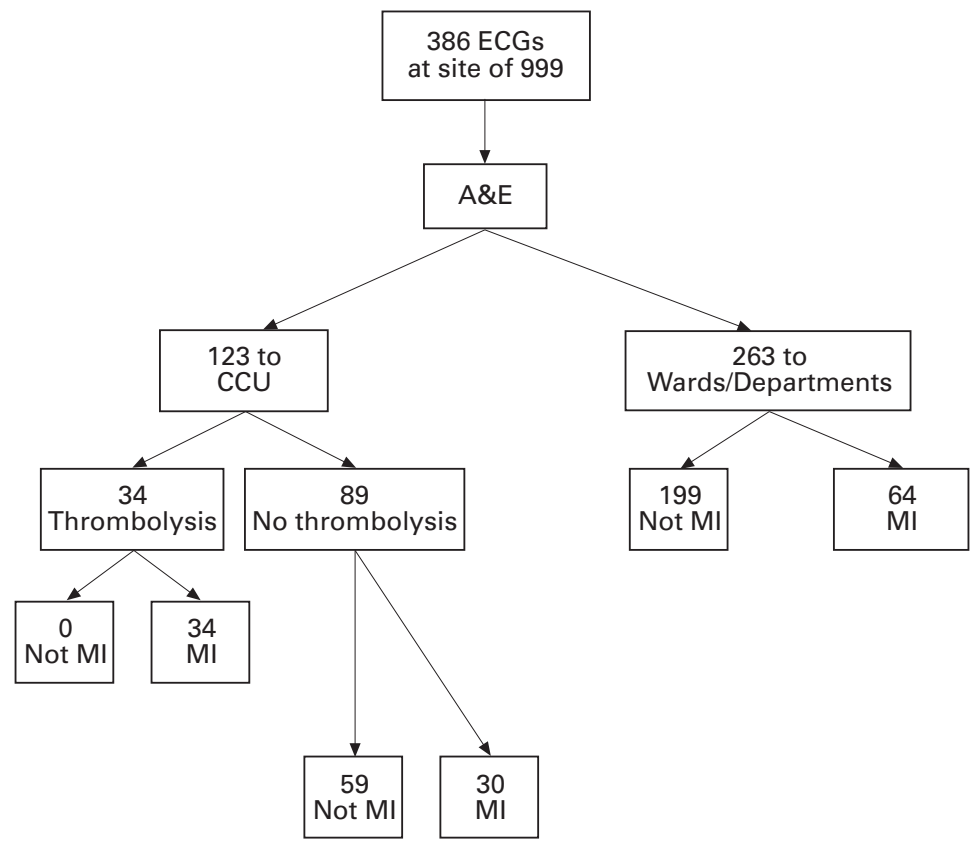

Figure 1 Distribution and management of patients in phase I.

patient directly to the CCU. Admission was arranged directly by VHF radio to the CCU, where the nurse in charge prepared a bed and contacted the admitting physician with details of the estimated arrival time. The physician was asked to attend the CCU before the arrival time. On arrival of the patient, if myocardial infarction was confirmed, thrombolytic treatment was started without delay, unless there were exclusion criteria. Patients with bundle branch block and clinical features of myocardial infarction were also given thrombolytics, and patients with ischaemic ST depression were treated with heparin, aspirin, and nitrate. If the paramedic found no evidence of myocardial infarction or ischaemia on the initial ECG then the patient was taken to the accident and emergency department for assessment by a casualty officer and appropriate management.

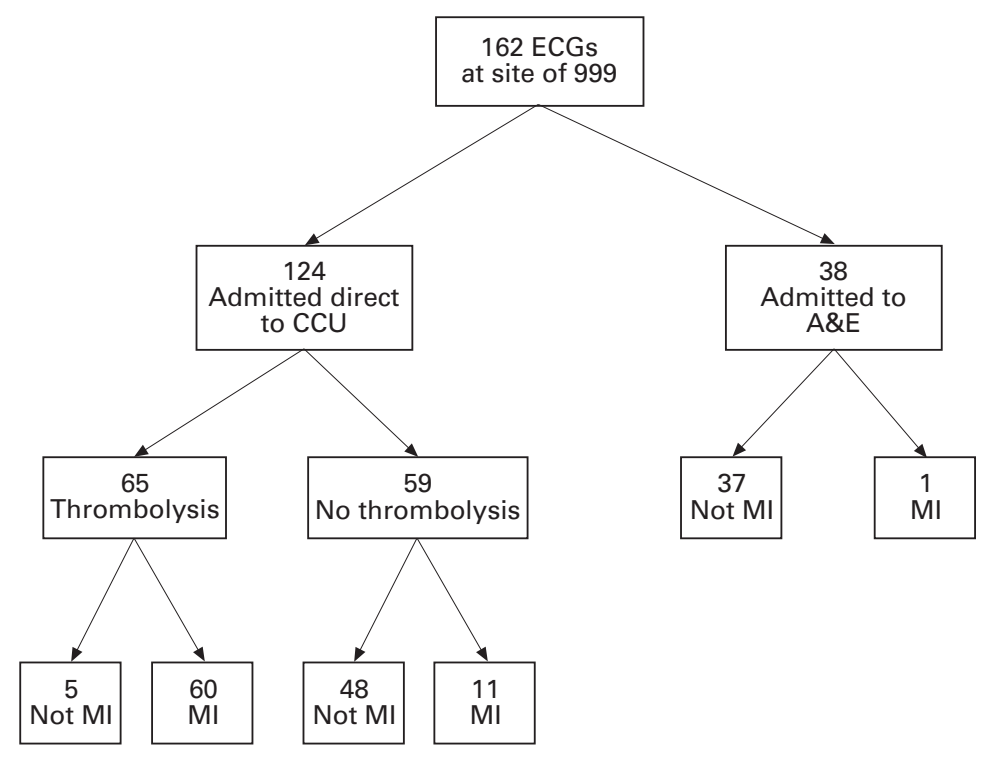

Figure 2 Distribution and management of patients in phase II.
During the study only paramedics involved in phase II were permitted to admit patients directly to the CCU, which was dependent on ECG interpretation by the paramedic. On arrival, the ECG was examined by the admitting physician and, if warranted, a further recording was made. Traces that were clear and unequivocal were acted on immediately.

The CCU at Derbyshire Royal Infirmary is an eight bed unit and the standard policy is always to have a bed available for an immediate acute admission. Patients are normally transferred to a general medical ward after 24-48 hours, or when clinically stable. During the study, detailed information on all interval durations, patient outcome, and paramedic ECG interpretation accuracy were documented.

The major cost of the study was that of additional paramedic training, which was costed at $£ 385$ per paramedic. Other expenses included the provision and installation of a VHF radio in coronary care and the availability of electrocardiographic equipment suitable for standard 12 lead recording for each paramedic ambulance.

Phase I of the study started in June 1992 and phase II in September 1993. The study was concluded in April 1995.

\section{Results}

During phase I, ECGs were recorded and reported on by the paramedics at the site of the emergency service call of 386 patients (mean age 66.8 (range 44-87) years; 293 men, 93 women) who contacted the emergency services directly with symptoms suggestive of myocardial infarction. These patients were taken to the accident and emergency department at the Derbyshire Royal Infirmary for assessment and appropriate management. Of the 64 patients who had a confirmed diagnosis of myocardial infarction, 53\% subsequently received thrombolytic treatment. Figure 1 shows the pathways followed by the patients in phase I.

During phase II ECGs of 162 patients with suspected myocardial infarction were recorded by the paramedics. A total of 124 patients (mean age 64.6 (range 37-87) years; 99 men, 25 women) were admitted directly to the CCU by the paramedic service. The thrombolysis rate for the 71 patients with confirmed myocardial infarction was $83 \%$. Figure 2 shows the pathways followed by these patients. Unstable angina was diagnosed in 35 patients. The remaining 38 patients were taken to the accident and emergency department because the initial ECG did not show evidence of either myocardial infarction or ischaemia. Of these, one was subsequently shown to have sustained a myocardial infarction.

All ECGs in both phases were subsequently graded by a cardiologist for technical quality and accuracy of interpretation. The average interpretation accuracy rate was $87.5 \%$ (range $80-100 \%$ for individual paramedics) in phase I and $92 \%$ in phase II. Despite often difficult circumstances and surroundings the technical quality of recorded ECGs was very satisfactory. A total of 323 ECGs (83.7\%) recorded in phase I were graded as acceptable or good and 


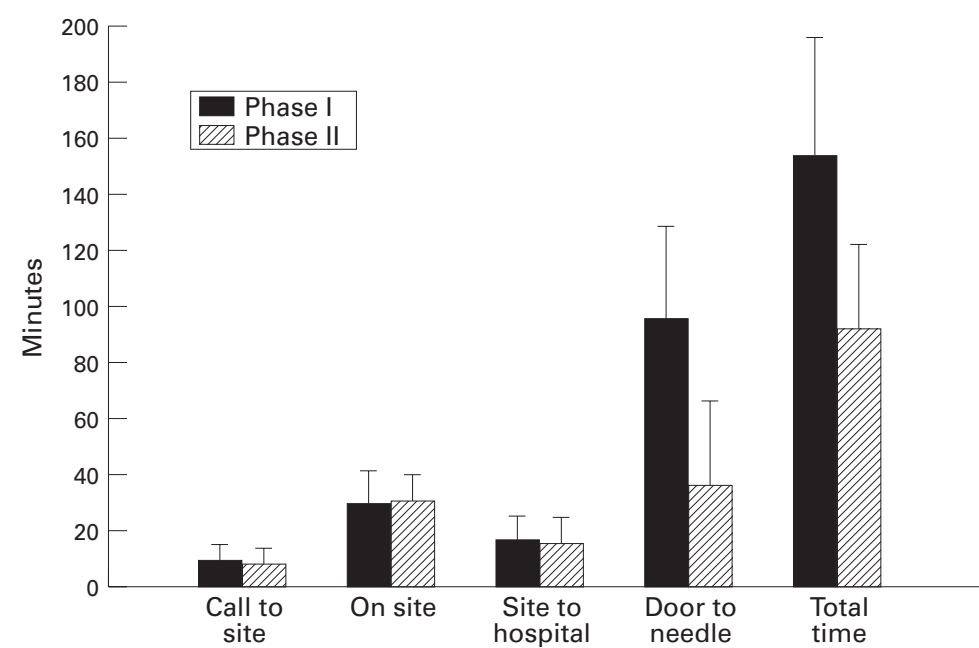

Figure 3 Comparison of mean delay times between phases I and II.

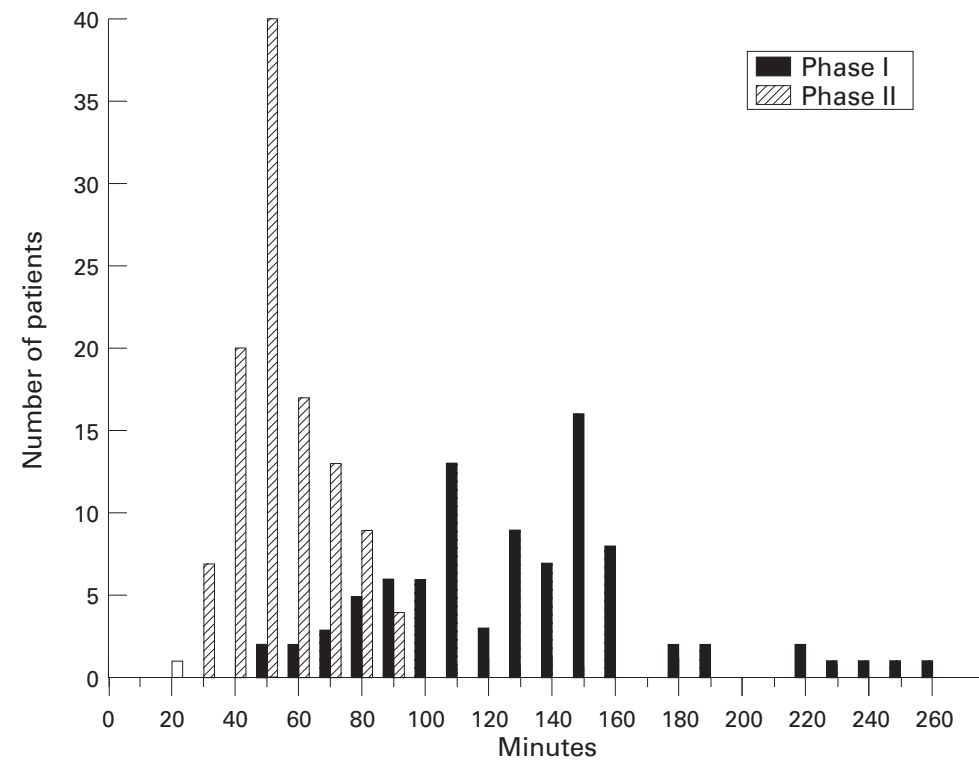

Figure 4 Time from call to emergency services to arrival in the coronary care unit.

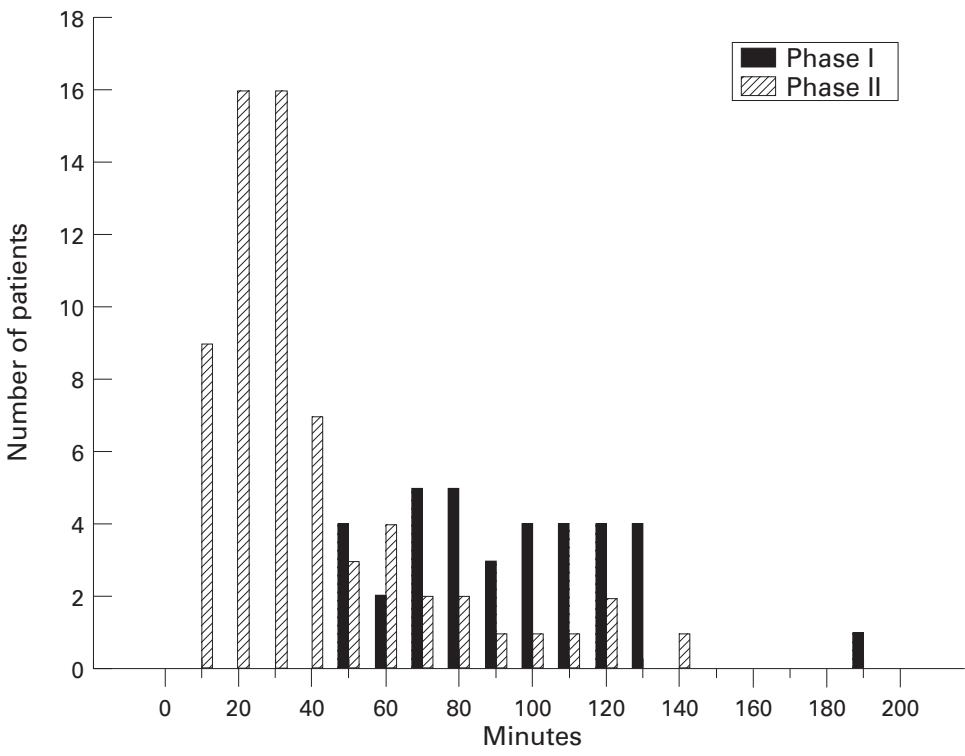

Figure 5 Door to needle time.
$150(92.6 \%)$ in phase II. All paramedics who entered phase I achieved satisfactory ECG recording and interpretation skills required for progression to phase II.

Comparison of the average interval durations in phases I and II was made using the Mann-Whitney U test (fig 3).

The call to needle interval for all patients receiving thrombolytic treatment was reduced from 154 to 93 minutes $(\mathrm{p}<0.001)$ and the hospital door to needle interval was reduced from 97 to 37 minutes $(p<0.001)$. The call to site of episode, on site, and site to hospital intervals were all similar. Figure 4 shows the call to CCU interval for all 123 phase I and 124 phase II patients who were admitted to the CCU, either through the emergency department (phase I) or directly by the paramedic service (phase II). Figure 5 shows the door to needle interval of the 34 patients in phase I and 65 patients in phase II who received thrombolytic treatment.

All distributions of interval durations for patients in phase II are skewed, and in the case of the distribution of door to needle intervals is attributed to "clinical" delay in the treatment of some patients. On each occasion, the patient with suspected myocardial infarction was seen immediately on arrival in the CCU by the admitting physician, and myocardial infarction was confirmed by ECG without attendant contraindications to thrombolysis in 41 patients. In this group the average door to needle interval was 19 (range 3-29) minutes. Thrombolysis was delayed in the remaining 24 patients by the admitting physician on clinical grounds. In 15 patients the ECG was not regarded as diagnostic, five were thought to have a significant bleeding risk, and two were hypertensive. One patient had had streptokinase for a previous episode, and delay ensued in establishing this fact and obtaining t-PA for the treatment of myocardial infarction. On one occasion the admitting physician was called to a cardiac arrest as the patient arrived and this resulted in a delay. Thrombolytic treatment was subsequently administered after an average of 58 (range 30-135) minutes in patients for whom there was a clinical reason for delay.

Thrombolysis was withheld in 11 patients with confirmed myocardial infarction in phase II by the admitting physician. Seven patients were thought to have non-diagnostic ECG changes, two had active peptic ulceration, one had severe hypertension, and one sustained a cardiac arrest as he arrived in the CCU (resuscitation was unsuccessful). Of the seven whose ECGs were considered to be non-diagnostic by the admitting physician, two had been admitted by the paramedics with an interpretation of probable myocardial infarction.

Four patients with symptoms suggestive of myocardial infarction whose ECGs showed bundle branch block were admitted in phase II. All four were thrombolysed and myocardial infarction was confirmed in three.

Myocardial infarction was excluded by serial ECGs and cardiac enzymes in 53 patients admitted directly to the CCU by paramedics in phase II. Forty four had ischaemic changes on 
the initial ECG and were treated on admission with heparin, nitrates, and $\beta$ blockers. Four patients were initially thought by the admitting physician to have diagnostic anteroseptal ST segment elevation, and received thrombolytic treatment. The remaining four were considered to have non-diagnostic ECG changes.

During phase II 38 patients were taken to the accident and emergency department because there were no diagnostic features on their ECGs. A diagnosis of non-cardiac chest pain was made in 35 , two were admitted to medical wards with a diagnosis of angina, and one was subsequently shown to have sustained an acute inferior myocardial infarction. In that patient, diagnostic changes were present on an ECG repeated in the emergency department one hour after the initial, non-diagnostic recording made by the paramedics. He was subsequently transferred to the CCU, thrombolysed and made an uncomplicated recovery.

While this study did not aim to assess the effect of the scheme on mortality, and numbers were not sufficiently great to afford statistical significance, the death rates seem to show a trend that is consistent with current expectations. In phase I there were 128 patients with confirmed myocardial infarctions, of whom 20 (15.6\%) died within 42 days. Of these, 14 died in transit to hospital, in the emergency department, and on medical or geriatric wards. The remaining six deaths were in patients transferred to the CCU. None of the deaths in phase I occurred in patients who received thrombolytic treatment. In phase II there were 71 patients with confirmed myocardial infarctions, of whom six $(8.4 \%)$ died within 42 days. One of these patients did not receive thrombolysis as his death occurred immediately on arrival in coronary care. The remaining five patients received thrombolytic treatment.

\section{Discussion}

This study has shown that the interval duration between a patient calling the emergency services and subsequently receiving thrombolytic treatment after ECG confirmation of myocardial infarction may be reduced by the provision of a specially trained paramedic service. Interval duration was reduced by early ECG diagnosis at the site of call with direct admission to coronary care, which bypassed the potential delay in the emergency department. Interval duration was also reduced by the paramedic team contacting the CCU directly by $\mathrm{VHF}$ radio and giving an estimated time of arrival, thereby avoiding delays caused by the ambulance controller relaying a message by telephone by the hospital switchboard. This allowed the CCU nursing staff to prepare a bed and to arrange for the admitting physician to be present before the patient's arrival. Arrival of the patient could then be followed quickly by clinical assessment of the patient by the admitting physician, ECG confirmation of myocardial infarction, and immediate administration of thrombolytic treatment prepared by the nursing staff on the unit. The mode of hospital admission has not been associated with administrative problems, but a coronary care bed policy has been instituted to ensure that a bed is always available for direct admission. We have not encountered any difficulties in training paramedics to record and interpret 12 lead ECGs, but we would emphasise that only experienced, fully qualified paramedics were invited to undergo advanced electrocardiographic training, and that they were assessed in detail individually before being allowed to enter phase II when diagnostic decisions were made. All patients with a normal or nondiagnostic ECG were transferred to the emergency department and paramedics were not authorised to make a decision that involved leaving the patient at the site of the call.

Although thrombolytic treatment has proven benefit in patients with myocardial infarction, all studies have shown a major complication rate of approximately $0.5 \%$ and it is generally agreed that such treatment should not be administered unless there is ECG confirmation of acute myocardial infarction. ${ }^{21}$ It is well accepted that diagnosis of myocardial infarction in the early stages is often difficult and that clinical diagnosis, without ECG, is often unreliable. ${ }^{22}$ Pre-hospital administration of thrombolytic treatment by general practitioners has major logistical implications regarding the availability of an ECG, appropriate resuscitation facilities, and drug storage, and it is unlikely that this will receive widespread acceptance, particularly as the number of acute myocardial infarctions seen annually by individual general practitioners is small. Potential bleeding complications of thrombolysis are such that administration by a paramedic team, acting independently, would have potential ethical and legal implications. This is particularly likely to occur in patients with acute aortic dissection, which sometimes mimics myocardial infarction. If thrombolytic treatment is to be administered in hospital rather than before admission, the use of a "fast track" to avoid delay and the possibility of administration in the emergency department before transfer to coronary care are of potential benefit. This may be appropriate in individual hospitals where the necessary resources are available and where the workload in the emergency department would allow such a system to operate. We believe, however, that it may be preferable for myocardial infarction to be confirmed before hospital arrival and the patient admitted directly to a coronary unit, thereby bypassing the emergency department completely.

Although paramedic ambulance services have been in existence for over 20 years, training has been directed largely towards resuscitation and emergency treatment, rather than potentially important ECG diagnosis after myocardial infarction despite the fact that this possibility has been suggested. ${ }^{23}$

Traditionally, patients have been instructed to contact their general practitioner after developing chest pain, and await a home visit with subsequent hospital admission. This is associated with considerable delay ${ }^{24}{ }^{25}$ compared with self admission by the emergency service. This has led to local recommendations for patients and general practitioners to contact 
the emergency service directly if symptoms suggest myocardial infarction, rather than arrange a home visit. We are not aware of any similar programme involving a district paramedical service recording and interpreting ECGs, although a service using a hospital based nurse/paramedic team has been used in conjunction with a rural community hospital. ${ }^{26}$

The objective of this study was to reduce the delay before thrombolytic treatment was administered, but the decision to use thrombolysis was made by the admitting medical staff in the CCU, not by the paramedic. The proportion of patients with a diagnosis of definite myocardial infarction who received thrombolysis in phase I was similar to that of all patients passing through the CCU during that time. This is supported by data held in audit files for patients admitted to the CCU, which have been kept at the Derbyshire Royal Infirmary since 1991. Although a parallel study could have been performed, comparing direct admission by ECG trained paramedics with direct accident and emergency transfer by the "standard" paramedic service, this was not feasible given the paramedic resources available, and the prospective study described was used. This had the advantage of using the earlier phase I data for the control group. However, neither the paramedics nor the coordinator of the study were aware of the time intervals or clinical outcome until the study was completed.

It is recognised that further investigation is desirable to confirm these findings. It is hoped that collaboration with other hospitals in the area will be possible to undertake a larger study. In conclusion, this study has shown that specialised training of paramedics to accurately record and interpret ECGs of patients with suspected myocardial infarction is feasible, and that it can lead to a reduction in the interval duration to thrombolytic treatment of almost one hour. Previous work has suggested that initiating thrombolysis 60 minutes earlier would save about 23 extra lives for every 1000 treated patients. ${ }^{27}$ Although lower mortality was not an end point of this study, there was encouraging evidence that reduced death rates could be achieved by a trained paramedic service, with early ECG recording and interpretation, direct coronary care admission, and a reduction in treatment delay, with little additional cost and at no risk to the patient, which may have important implications for the early management of myocardial infarction.

This paper was presented at the annual meeting of the American College of Cardiology in Orlando, USA, 1996.

This project was funded by the NHS, including a small gran from district audit funds. We gratefully acknowledge the hard work and commitment of the paramedics who participated, without whom the study would not have been possible: $M$ Barnet, T Barton, D Birkenshaw, B Blood, M Conibear, P Fitzsimmons, M Foster, M Haslam, C Hepworth, C Hill, R Hill, S
Holden, G Howard, V Lancashire, B Peacock, R Ratcliffe, C Reid, A Rippon, C Roberts, M Smith, K Sykes, C Tempest, M Towers, $\mathrm{P}$ Wheeldon, $\mathrm{D}$ Wiggins, $\mathrm{M}$ Wiggins, and $\mathrm{R}$ Winfield. We are also thank Dr Lesley Rushton, Department of Public Health Medicine and Epidemiology, Queen's Medical Centre, Nottingham, for statistical advice, and Physio Control Ltd, Bas- ingstoke, and Marquette Electronics, Manchester, for the loan of electrocardiographic recording equipment.

1 GISSI (Gruppo Italiano per lo Studio della Streptochinasi nell 'infarcto miocardico). Effectiveness of intravenous thrombolytic treatment in acute myocardial infarction. Lancet 1986;i:397-401.

2 ISIS-2 (Second International Study of Infarct Survival) Collaborative Group. Randomised trial of intravenous streptokinase, oral aspirin, both, or neither among 17,187 cases of suspected acute myocardial infarction. Lancet 1988;ii:349-60.

3 Fibrinolytic Therapy Trialists' Collaborative Group. Indications for fibrinolytic therapy in suspected acute myocardial infarction: collaborative overview of early mortality and major morbidity results from all randomised trials of more major morbidity results from all randomised 1000 patients. Lancet 1994;343:311-22.

4 McMurray J, Rankin A. Treatment of myocardial infarction, unstable angina and angina pectoris. BMF 1994;309:134350 .

5 Kereiakes DJ, Weaver WD, Anderson JL, Feldman T, Gibler $\mathrm{B}$, Aufderheide $\mathrm{T}$, et al. Time delays in the diagnosis and treatment of acute myocardial infarction: a tale of eight cities. Am Heart F 1990;120:773-80.

6 Birkhead JS. Time delays in the provision of thrombolytic treatment in six district hospitals. BMF 1992;305:445-8.

7 Annual report of the director of public health. Derby: Southern Derbyshire Health Authority, 1991:17.

8 Rawles J on behalf of the GREAT group. Halving of mortality at 1 year by domiciliary thrombolysis in the Grampian region early anistreplase trial (GREAT). $7 \mathrm{Am}$ Coll Cardiol 1994;23:1-5.

9 Leizorowicz A, Boissel JP, Julian D, Castaigne A, Haugh MC (the European Myocardial Infarction Project Group). Prehospital thrombolytic therapy in patients with suspected acute myocardial infarction. $N$ Engl $f \mathrm{Med}$ 1993;329:283-9.

10 Weaver D, Cerqueira M, Hallstrom AP, Litwin PE, Martin JS, Kudenchuk PJ, et al. Prehospital-initiated vs hospitalinitiated thrombolytic therapy: the myocardial infarction triage and intervention trial. $\mathcal{F} A M A$ 1993;270:1211-16.

1 Sharkey SW, Brunette DD, Ruiz E, Hession WT, Wysham $\mathrm{DG}$, Goldenberg IF, et al. An analysis of time delays preceding thrombolysis for acute myocardial infarction. $\mathscr{F} A M A$ 1989;262:3171-4.

12 Pell ACH, Miller HC, Robertson CE, Fox KAA. Effects of "fast track" admission for acute myocardial infarction on delay to thrombolysis. BMF 1992;304:83-7.

13 Al-Mohammad A, Fath-Ordoubadi F, Amadi A, Beatt KJ. Can the pressure to achieve short door to needle time lead to inappropriate thrombolysis? [abstract] Br Heart f 1995; 73(suppl):P48.

14 Applebaum D, Weiss AT, Koren G, David YB, Hasin Y, Gotsman MS. Feasibility of prehospital fibrinolytic therapy in acute myocardial infarction. Am f Emerg Med 1986;4: 201-4.

15 Roth A, Barbash GI, Hod H, Miller HI, Rath S, Modin M, et al. Should thrombolytic therapy be administered in the mobile intensive care unit in patients with evolving myocardial infarction? f Am Coll Cardiol 1990;15:932-6.

16 Pantridge JF, Geddes JS. A mobile intensive care unit in the management of myocardial infarction. Lancet 1967;ii:271-3.

7 White NM, Parker WS, Binning RA, Kimber ER, Ead HW, Chamberlain DA. Mobile coronary care provided by ambulance personnel. BMF 1973;iii:618-22

18 Briggs RS, Brown PM, Crabb ME, Cox TJ, Ead HW, Hawkes RA, et al. The Brighton resuscitation ambulances: a continuing experiment in prehospital care by ambulance a continuing experiment in

19 Aufderheide TP, Hendley GE, Woo J, Lawrence S, Valley V, Teichman SL. A prospective evaluation of prehospital 12-lead ECG application in chest pain patients. $\mathcal{F}$ Electrocardiol 1992;24(suppl):8-13.

20 Sherringham P. Cellular transmission of 12-lead ECGs from a London ambulance vehicle. Ambulance UK 1992;7:6-9.

21 Weston CFM, Penn WJ, Julian DG (on behalf of the British Heart Foundation Working Group). Guidelines for the early management of patients with myocardial infarction. BMF 1994;308:767-71.

22 Rule S, Brooksby P, Sanderson J. Use of thrombolysis for acute myocardial infarction by general practitioners. Postgrad Med $\mathcal{F}$ 1993;69:190-3.

23 Cummins RO. From pain to reperfusion: what role for the prehospital 12-lead ECG? Ann Emerg Med 1990;19:1342-6. 4 Ahmad RAS, Bond S, Burke J, Singh SP, Watson RDS. Patients with suspected myocardial infarction: effect of mode of referral on admission time to a coronary care unit. Br F Gen Pract 1992;42:145-8.

25 Parry G, Wrightson WN, Hood L, Adams PC, Reid DS. Delays to thrombolysis in the treatment of myocardial infarction. $\mathcal{F}$ R Coll Physicians Lond 1993;27:19-23.

26 Foster DB, Dufendach JH, Barkdoll CM, Mitchell BK. Prehospital recognition of AMI using independent nurse/ paramedic 12-lead ECG evaluation: impact on in-hospital times to thrombolysis in a rural community hospital. $A m \mathcal{F}$ Emerg Med 1994;12:25-31.

27 Rawles J. Treatment of myocardial infarction and angina [letter]. BMF 1995;310:465. 